IRA-International Journal of Technology \& Engineering

ISSN 2455-4480; Vol.09, Issue 01 (October 2017)

Pg. no. 1-9

Institute of Research Advances

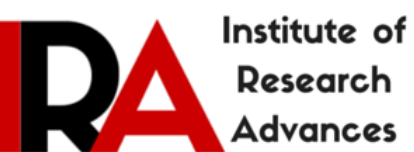

https://research-advances.org/index.php/IRAJTE

\title{
Automatic Analysis and Telemetry of Patients Affected by COPD
}

\author{
N. Adhithiya ${ }^{1} \&$ Dr. (Mrs). R. Sukanesh ${ }^{2}$ \\ ${ }^{1}$ Student, M.E.-Wireless Technologies, Thiagarajar College of Engineering, Madurai, India. \\ ${ }^{2}$ Professor and Head, Department of ECE, Thiagarajar College of Engineering, Madurai, India.
}

Type of Review: Peer Reviewed.

DOI: http://dx.doi.org/10.21013/jte.v9.n1.p1

\section{How to cite this paper:}

Adhithiya, N., Sukanesh, R. (2017). Automatic Analysis and Telemetry of Patients Affected by COPD. IRA International Journal of Technology \& Engineering (ISSN 2455-4480), 9(1), 1-9. doi:http://dx.doi.org/10.21013/jte.v9.n1.p1

(C) Institute of Research Advances.

(cc) EY-NC

This work is licensed under a Creative Commons Attribution-Non Commercial 4.0 International License subject to proper citation to the publication source of the work.

Disclaimer: The scholarly papers as reviewed and published by the Institute of Research Advances (IRA) are the views and opinions of their respective authors and are not the views or opinions of the IRA. The IRA disclaims of any harm or loss caused due to the published content to any party.

Institute of Research Advances is an institutional publisher member of Publishers Inter Linking Association Inc. (PILA-CrossRef), USA. The institute is an institutional signatory to the Budapest Open Access Initiative, Hungary advocating the open access of scientific and scholarly knowledge. The Institute is a registered content provider under Open Access Initiative Protocol for Metadata Harvesting (OAI-PMH).

The journal is indexed \& included in WorldCat Discovery Service (USA), CrossRef Metadata Search (USA), WorldCat (USA), OCLC (USA), Open J-Gate (India), EZB (Germany) Scilit (Switzerland), Airiti (China), Bielefeld Academic Search Engine (BASE) of Bielefeld University, Germany, PKP Index of Simon Fraser University, Canada. 


\begin{abstract}
Chronic Obstructive Pulmonary Disease (COPD) is an umbrella term for a number of diseases including emphysema, bronchitis and asthma and so on. The severity of the disease can be identified by finding the peripheral oxygen saturation (SpO2) level. This level can be decomposed into trend components to analyse the deterioration in condition of patients affected by COPD. The condition can be analysed on daily, weekly and monthly basis. Message is sent to the clinical attendant of the patient when the condition becomes very abnormal.
\end{abstract}

Keywords: COPD, peripheral oxygen saturation level, trend components, alert message

\title{
Introduction
}

The increase in pollution leads to malfunctioning of lungs. COPD is an indication of a number of lung disorders. Bronchitis is increase in mucus and inflammation. Emphysema is a chronic respiratory disease where there is over inflation of the air sacs or the alveoli in the lungs, causing a decrease in the lung function. Asthma is a condition in which a person's airways become inflamed, narrow and swell and produce extra mucus, which makes it difficult to breathe. In all these circumstances the main issue faced is breathlessness. This breathlessness leads to decrease in the peripheral oxygen saturation level usually termed as SpO2 level. This level tells the percentage of oxygenated hemoglobin when compared to the total amount of hemoglobin present in the blood.

$$
\text { Sp02 level }(\text { in } \%)=\frac{\begin{array}{c}
\text { Amount of oxygenated } \\
\text { heamoglobin }
\end{array}}{\text { Total amount of heamoglobin in blood }} * 100
$$

This breathlessness can't be cured but can only be treated and kept under control. To maintain the condition of the patient, the patient must be continuously monitored. The patient can be monitored by means of measuring the $\mathrm{SpO}_{2}$ level using a pulse oxiometer [3][7]. But this method is inefficient since it is not possible to monitor every patient manually. Thus automatic monitoring of health condition of patients becomes essential. The obtained time series $\mathrm{SpO}_{2}$ data from the patients are decomposed into trend components and are analysed.

\section{Methodology}

Our proposed system works with input data from the pulse oxiometer [3][7] which is a non-invasive method causing minimum discomfort to the patient. This input is decomposed into trend components by various smoothing techniques. After this decomposition, the abnormality of the patients is checked by using the residue component. If the abnormality exists, the alarm signal is generated as in [2] and alert message is sent to the clinical attendant by using [6][7][1]. The flow chart of the methodology is shown in Fig1.

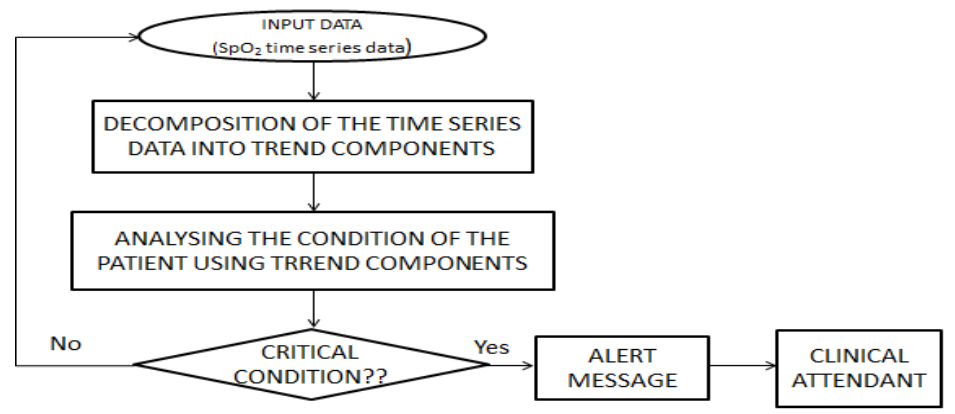

Fig.1: Detailed flowchart of the system. 


\section{Data Acquisition}

The acquisition of data is the main part of any analysis. The data acquisition can originally be done by using a pulse oxiometer. The pulse oxiometer is a device capable of measuring the peripheral oxygen saturation level of the patient affected by COPD [7]. The device can be placed inside the room where the patients are accommodated. The patients may be asked to take measurements of their oxygen saturation level twice a day, both in the morning and in the evening. This data can be used for analysis and decomposition. Since only a prototype is developed recorded data is used in this analysis. The patient whose $\mathrm{SpO}_{2}$ value is recorded died during the $134^{\text {th }}$ day of monitoring. This can be taken as the reference for verifying the correctness of the analysis.

\section{Data Decomposition}

The time series data is decomposed into two major components: short term trend and residual component [5]. The data decomposition is given in (1).

$$
\mathrm{s}_{\mathrm{i}}=\mathrm{m}_{\mathrm{i}}+\mathrm{r}_{\mathrm{i}} \quad(1)
$$

Short term trend essentially implies the condition of the patient for the past one week or two weeks. This can be used to determine if the patient's condition is improving or deteriorating for duration of 7 or 15 days. Long term trend is used to find the condition of the patient for duration of 60 to 90 days. The residue implies the day to day variation in the patient's condition.

\section{A. Calculation of short term trend:}

The short term trend is used to find if the health condition has deteriorated or improved over a period of 7 or 15 days. This calculation can be done by using simple symmetric moving average filter. Since the time series data is entered daily, asymmetric moving average filter will also give the same result.

$$
\mathrm{m}_{\mathrm{i}}=\left(\frac{1}{\mathrm{~N}}\right) \sum_{j=-k}^{k}(s i-j)
$$

$$
(\mathrm{k}=7 \text { or } 15)
$$

where $\mathrm{N}$ is the window size(7 or 15 in this case). There are differences in the type of window used for calculating short term trend. When seven-point window is used, the short-term trend closely follows the time series data. This means that this will clearly reveal the condition of the patient. If 15 -point window is used, the curve will be smoother. Other than this, there is one main advantage of increasing the window size. There may be missing readings due to the negligence of the patients in recording their $\mathrm{SpO}_{2}$ value. The 15-point window size is insensitive to these missing readings. This happens because of the increase in window size. But increasing the window size beyond this limit may lead to less accuracy in predicting the condition of the patient.

\section{B. Calculation of long term trend}

The long term trend is a quantitative measure of variation in the condition of the patient for duration of one month or above. For measuring this component also, the same moving average filter can be used with an increased window size. The moving average filter will be as in (2).

$$
\begin{aligned}
\mathrm{m}_{\mathrm{i}} & =\left(\frac{1}{\mathrm{~N}}\right) \sum_{j=-k}^{k}(s i-j) \\
\text { where } \mathrm{k} & =60 \text { or } 90
\end{aligned}
$$

As specified earlier, the curve will become very smooth but the curve will not follow the time series data. This smooth curve will fetch us the information about the condition of the patient. If the curve decreases, it means that the condition is deteriorating and if it increases, there is an improvement in the treatment given to the patient. Curve fitting techniques can also be used to find the long term trend. Techniques like exponential fitting, linear polynomial fitting can be used to fit the time series data into a curve. 
Curve smoothing techniques can also be used to fit the time series data. Fitting the data is essential in order to know the direction in which the patient's condition has turned after treatment.

The most popular technique is the least square regression technique. LOWESS is the popular curve fitting technique. LOWESS (Locally Weighted Scatterplot Smoothing)s is a popular tool used in regression analysis that creates a smooth line through a time plot or scatter plot to help you to see relationship between variables and foresee trends. LOESS is called as locally weighted scatter plot which is a variant of LOWESS.The LOWESS method and robust version of LOWESS curve smoothing technique can yield significant result in the estimation of long term trend. LOWESS is better than robust LOWESS technique. The robust version assigns lower weight to data that are far outside the curve. This will not give fair estimation of trend function since outliers are more important for estimation when compared to the middle points. The LOESS curve uses quadratic polynomial for smoothing the data points in a curve. The robust version of LOESS has the same problem of assigning lower weights to outliers.

Though there are different methods for computing the long term trend, there is no computational restriction as to which method must be used. The moving average filter is simple and requires less time when compared to the Least Square regression method adopted for smoothing.

C. Calculation of residue:

The residue function is used to calculate the day to day fluctuation in the patient's condition. This can be used to find any sudden change in the patient's condition from previous day to the current day. This can be found be finding the difference between the estimate of the short term trend and the actual reading of the day. The properties of the residues will be similar to noise. It has zero mean and it is a stationary process. Theresidue can be calculated using the formula (3).

$$
\mathrm{r}_{\mathrm{i}}=\mathrm{m}_{\mathrm{i}} \sim \mathrm{S}_{\mathrm{i}}
$$

Thus the standard deviation over the short term trend and the variance can imply the physiological condition of the patient over duration of 60 or 90 days.

The standard deviation of the residue over the short term trend can also be used as a measure to determine the day to day variation in the patient's condition.

\section{Results}

The $\mathrm{SpO}_{2}$ time series data is completely random in nature. The data is a stationary process which means that the pdf at various time intervals are strictly uncorrelated. The database details obtained had the following details.

i. The patient had pulmonary fibrosis.

ii. Oxygen therapy was given to the patient from day 34 .

iii. The patient died on the 135-th day of treatment.

The data is obtained by keeping a pulse oxiometer for measuring the $\mathrm{SpO}_{2}$ time series data twice a day. Due to age factor, there are missing data in the dataset. The first calculation is the calculation of short term trend by using moving average filter with a window size of 7 or 15 . The results are shown in fig. 2 . 


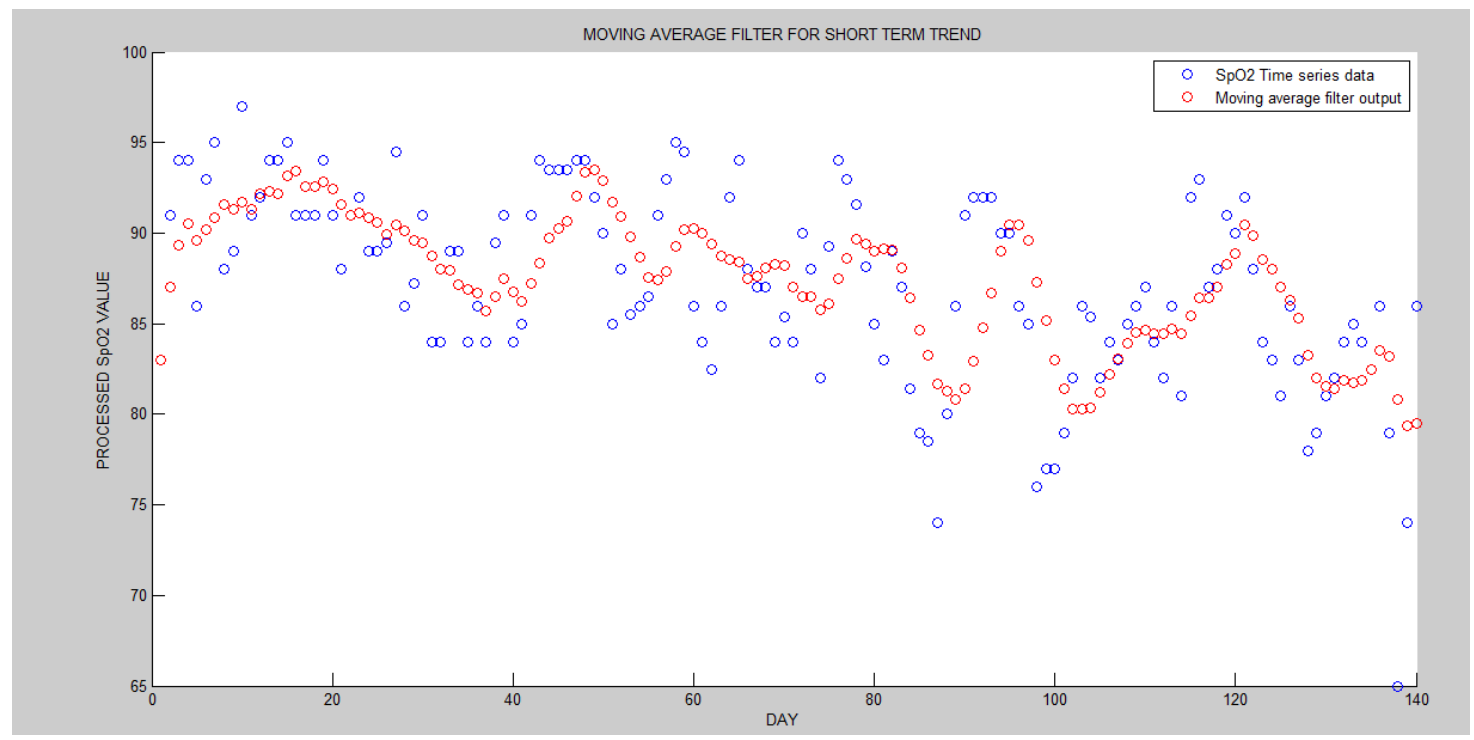

Fig2: Scatter Plot: $\mathrm{SpO}_{2}$ time series data-scatter plot. 7-point moving average filter output.

As said earlier, the 7-point moving average results closely follows the obtained time-series data. But analysing the trend is a bit difficult since the time series data and in turn the short term trend component obtained using the moving average filters are completely random in nature. The same short-term trend can also be calculated by using 15 point moving average filter. The results are shown in fig. 3 .

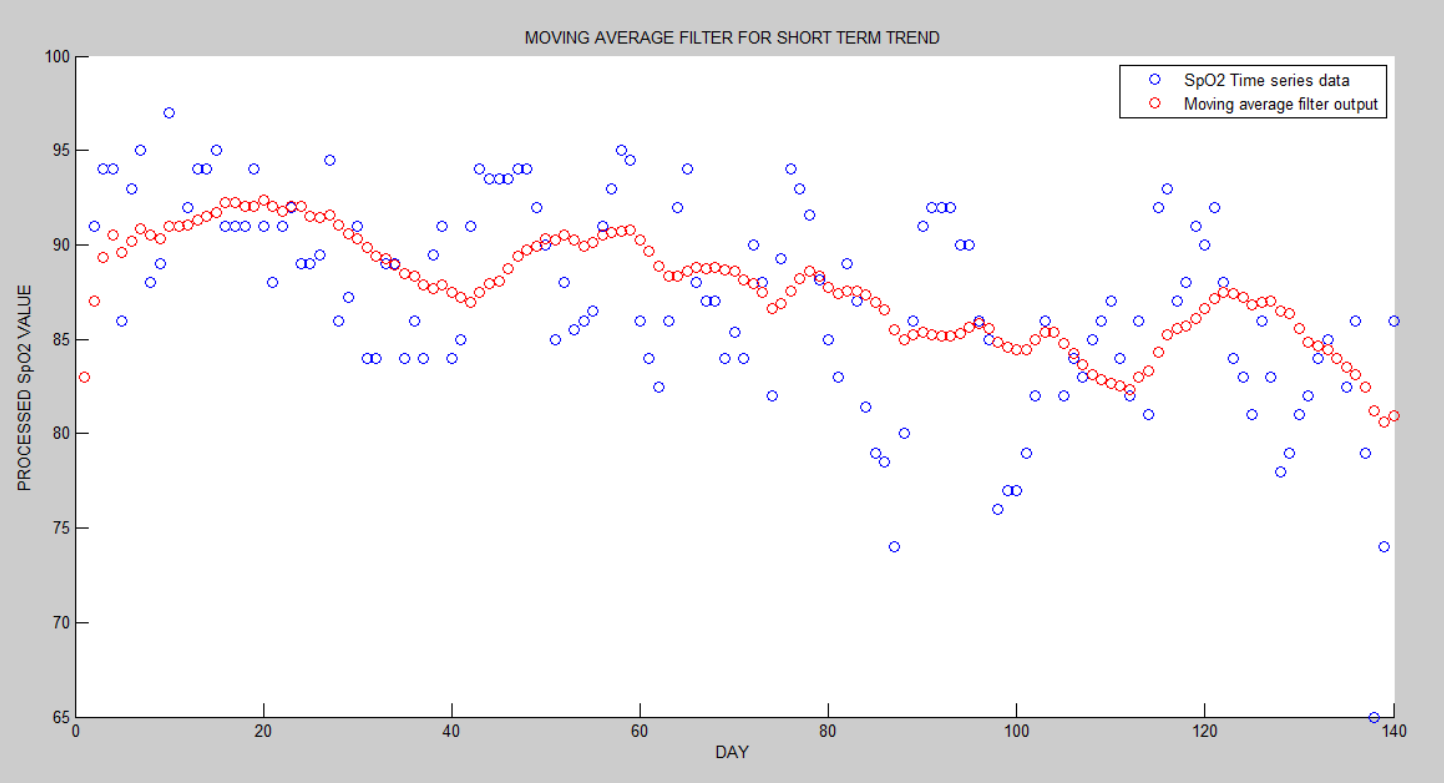

Fig.3: Scatter Plot: $\mathrm{SpO}_{2}$ time series data-scatter plot. 15-point moving average filter output.

As said earlier, the curve becomes smoother. But the moving average results did not follow the input time series data exactly. The 15-point window is insensitive to missed readings. From this result it is very clear that the condition of the patient has deteriorated rather than improving. On further improving the window size to 31 the results become inaccurate. This can be proved in the result obtained using the 31-point window as shown in fig. 4 . 


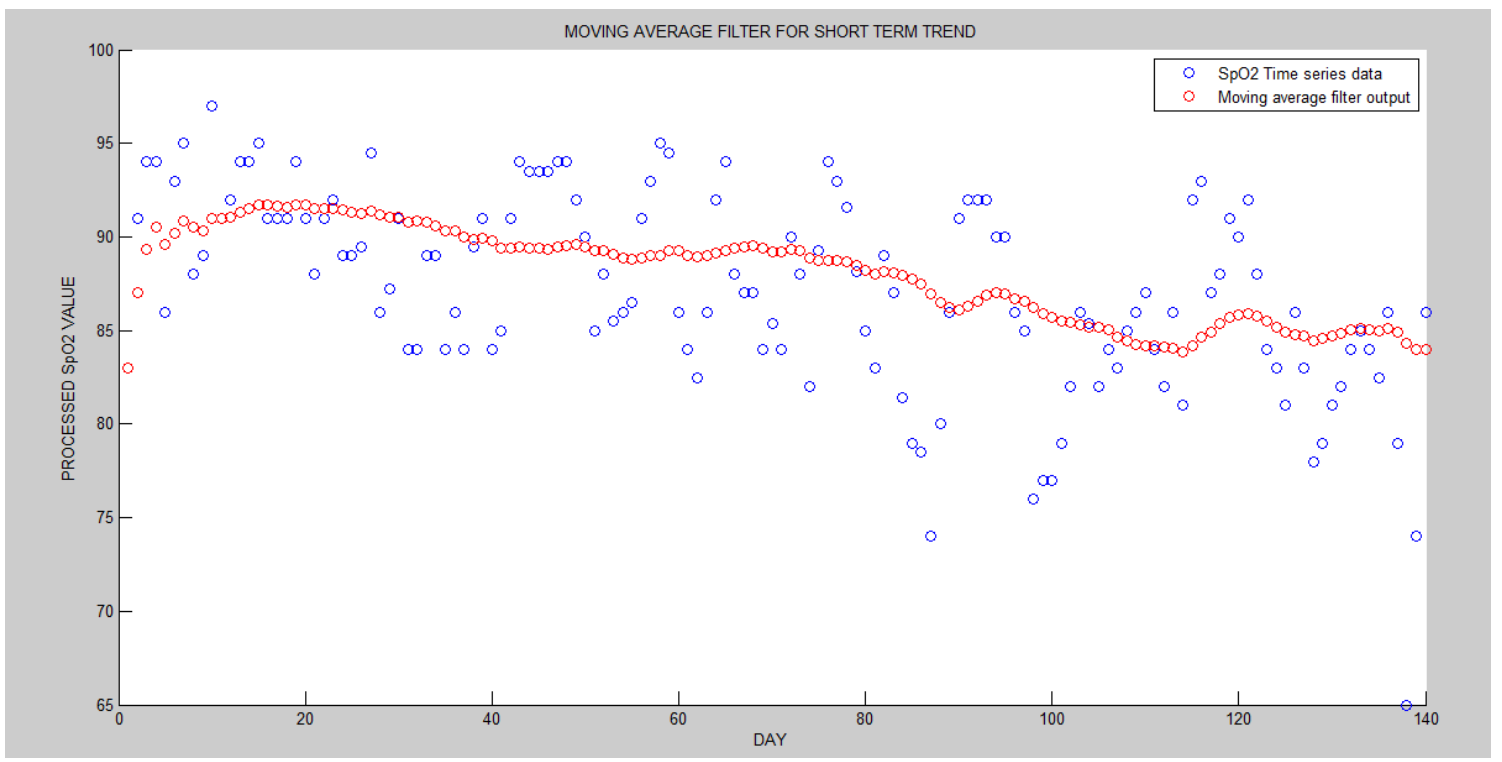

Fig.4: Scatter Plot:SpO $\mathrm{S}_{2}$ time series data-scatter plot. 31-point moving average filter output.

The deterioration of the condition during the fourth week of observation is not visible in this trend function analysis. Thus increasing the window size beyond 15 can't produce favourable results.

The long term trend calculated using the moving average filter with increased window size of 60 and 90 depicts the condition of the patient for a longer duration of a month or two. The trend will be almost linear showing the gradual deterioration in the condition of the patient. The curve becomes very smoother as the window size is increased largely. The moving average filter results are shown in fig.5 and fig.6.

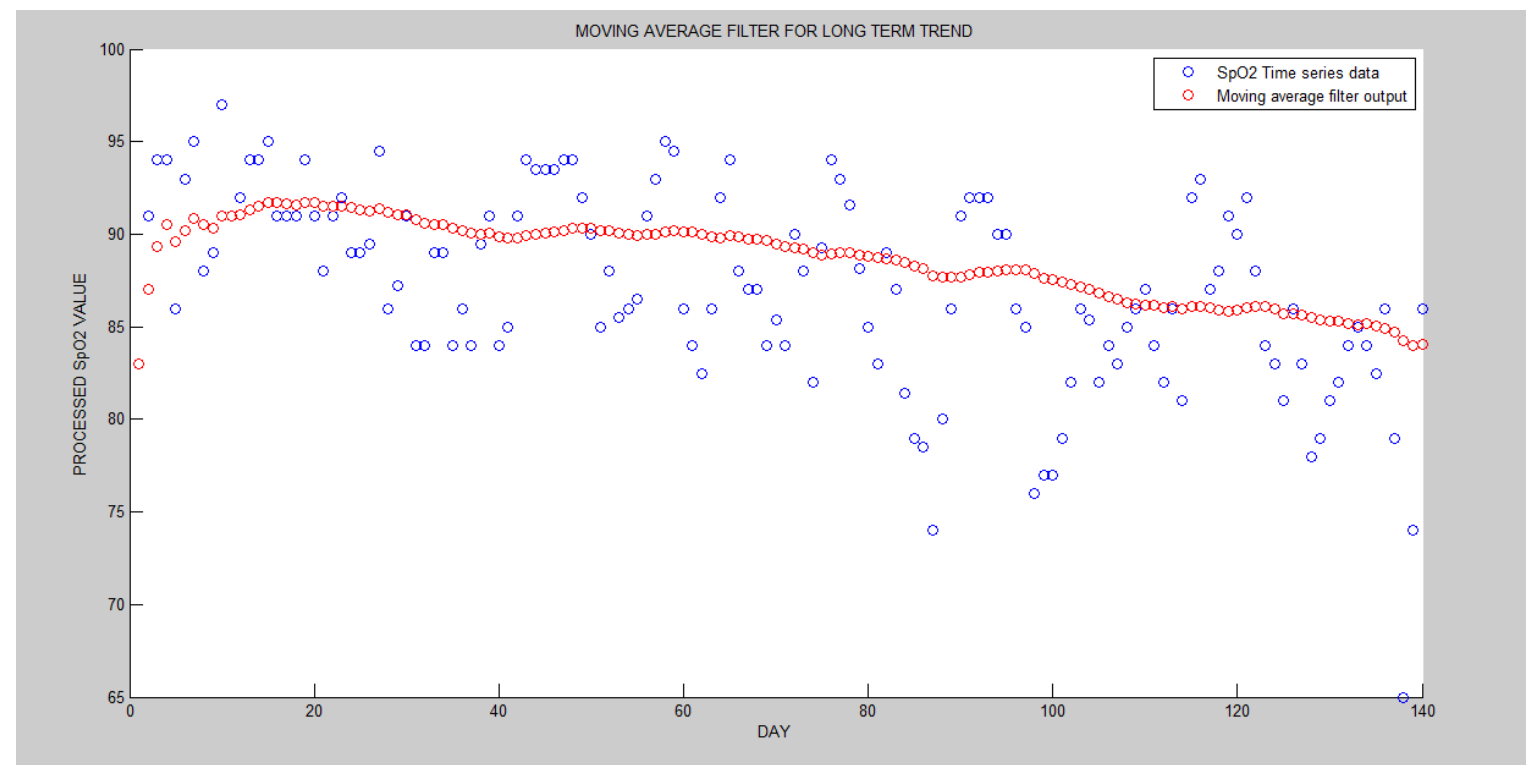

Fig5: Scatter Plot: $\mathrm{SpO}_{2}$ time series data-scatter plot. 60-point moving average filter output. 


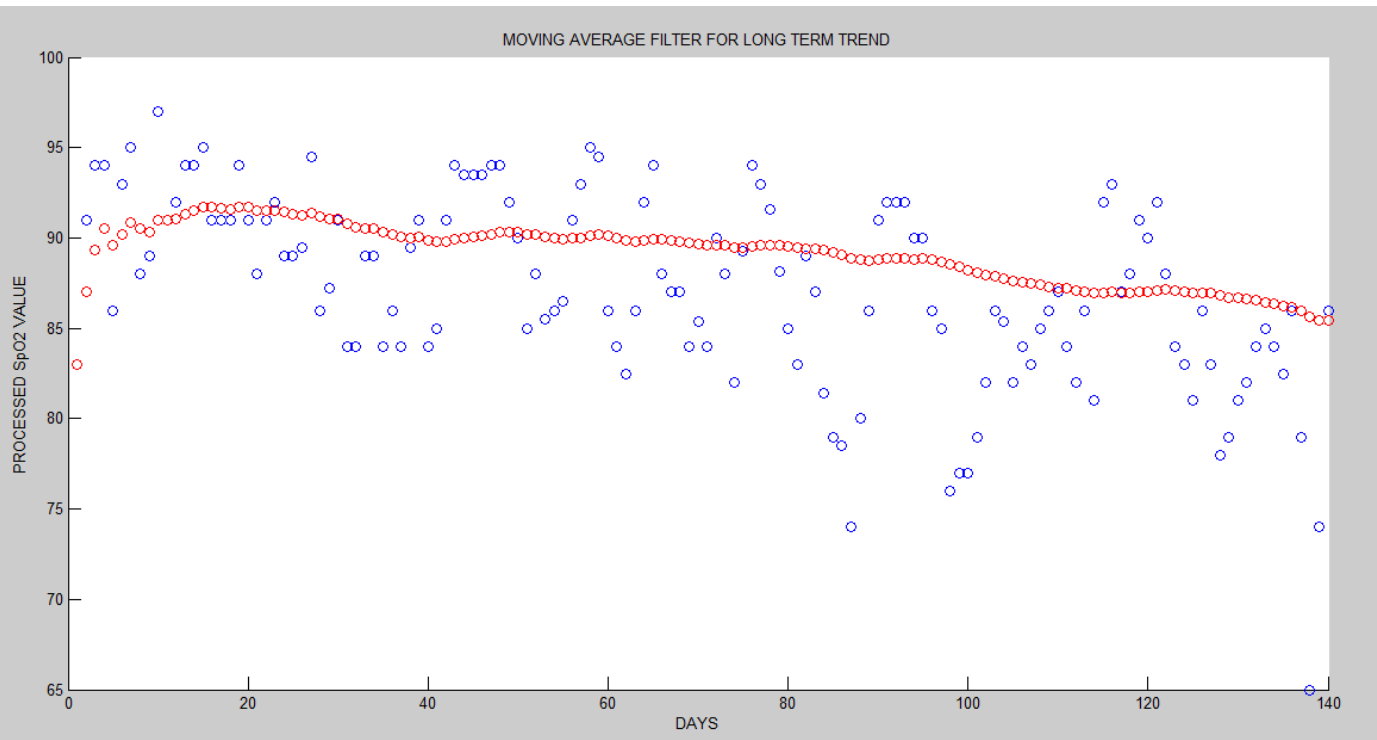

Fig.6: Scatter Plot: $\mathrm{SpO}_{2}$ time series data-scatter plot. 90-point moving average filter output.

As described earlier, the robust version of LOWESS is poor when compared to normal least square regression technique. The problem of assigning lower priority to outliers make r-LOWESS worse when compared to LOWESS. The LOWESS and r-LOWESS curve smoothing is shown in fig.7.

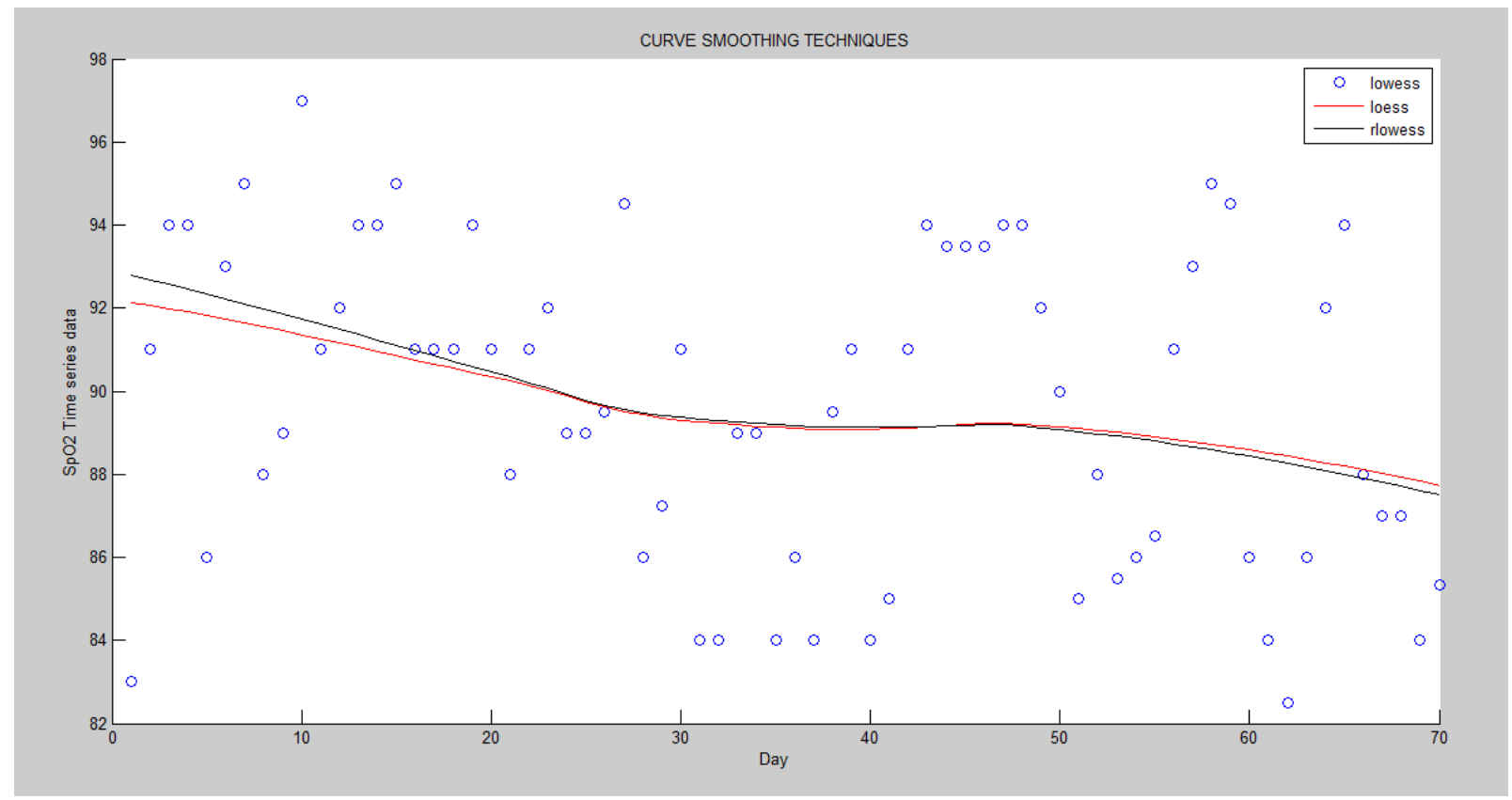

Fig7. Scatter Plot: $\mathrm{SpO}_{2}$ time series data-scatter plot.LOWESS and r-LOWESS smoothing of the input data for long term trend calculation.

In the same way, the LOESS technique can also fetch good results by using a second order polynomial. But the robust version of LOESS is not efficient. The curve of LOESS and r-LOESS is shown in fig.8. 


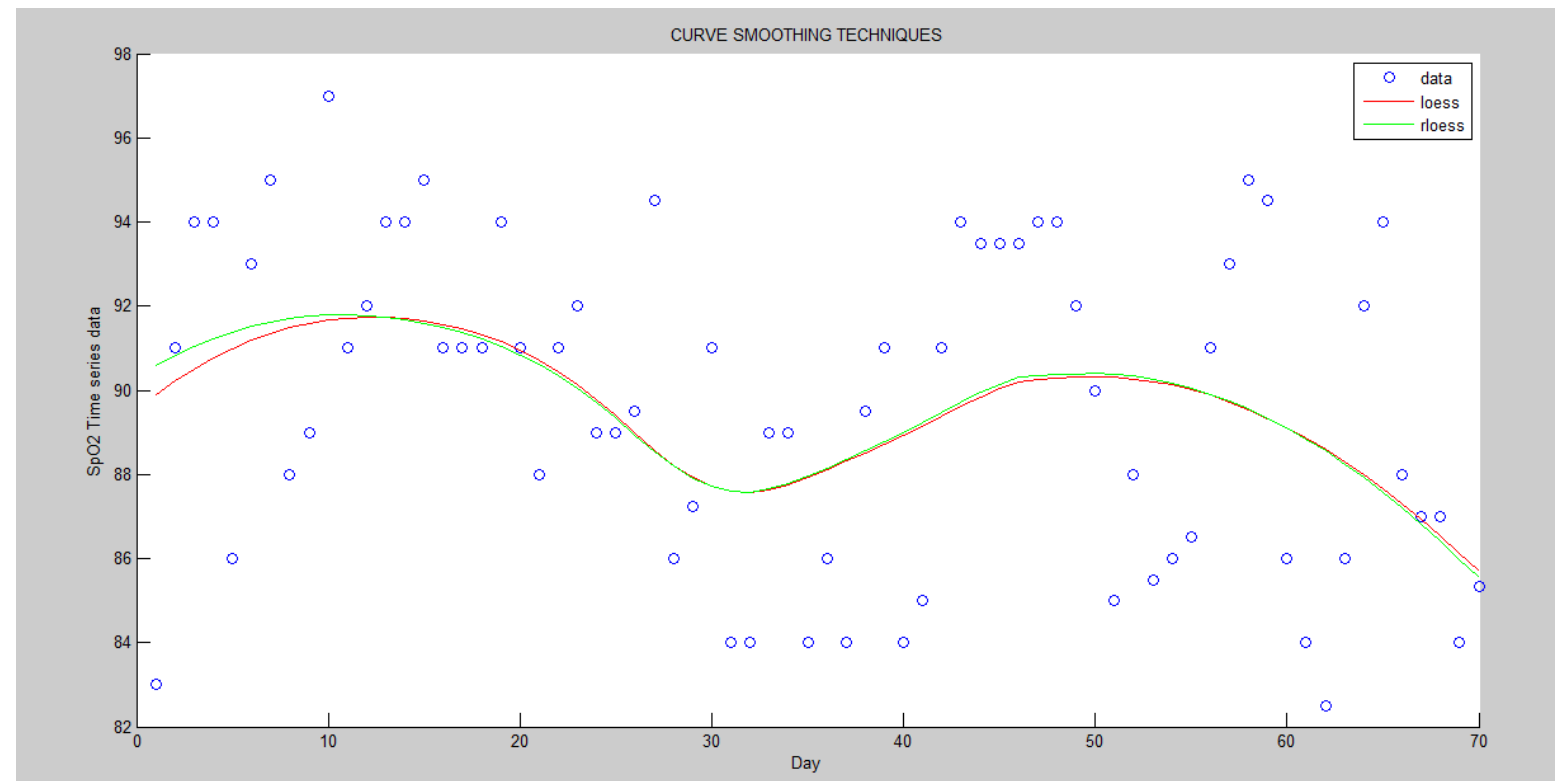

Fig.8: Scatter Plot: $\mathrm{SpO}_{2}$ time series data-scatter plot. LOESS and r-LOESS smoothing of the input data for long term trend calculation.

Out of these four smoothing techniques, experimental results show that LOWESS is the best way of depicting the condition of the patient.

The residue calculation is the important part of the analysis. This indicates the day to day variation in the condition of the patient. This will be greatly helpful in the estimation of critical condition of the patient and transmission of alarm signal. The residue is completely random in nature since the time series data is random. The residue of the time series data is shown in fig.9.

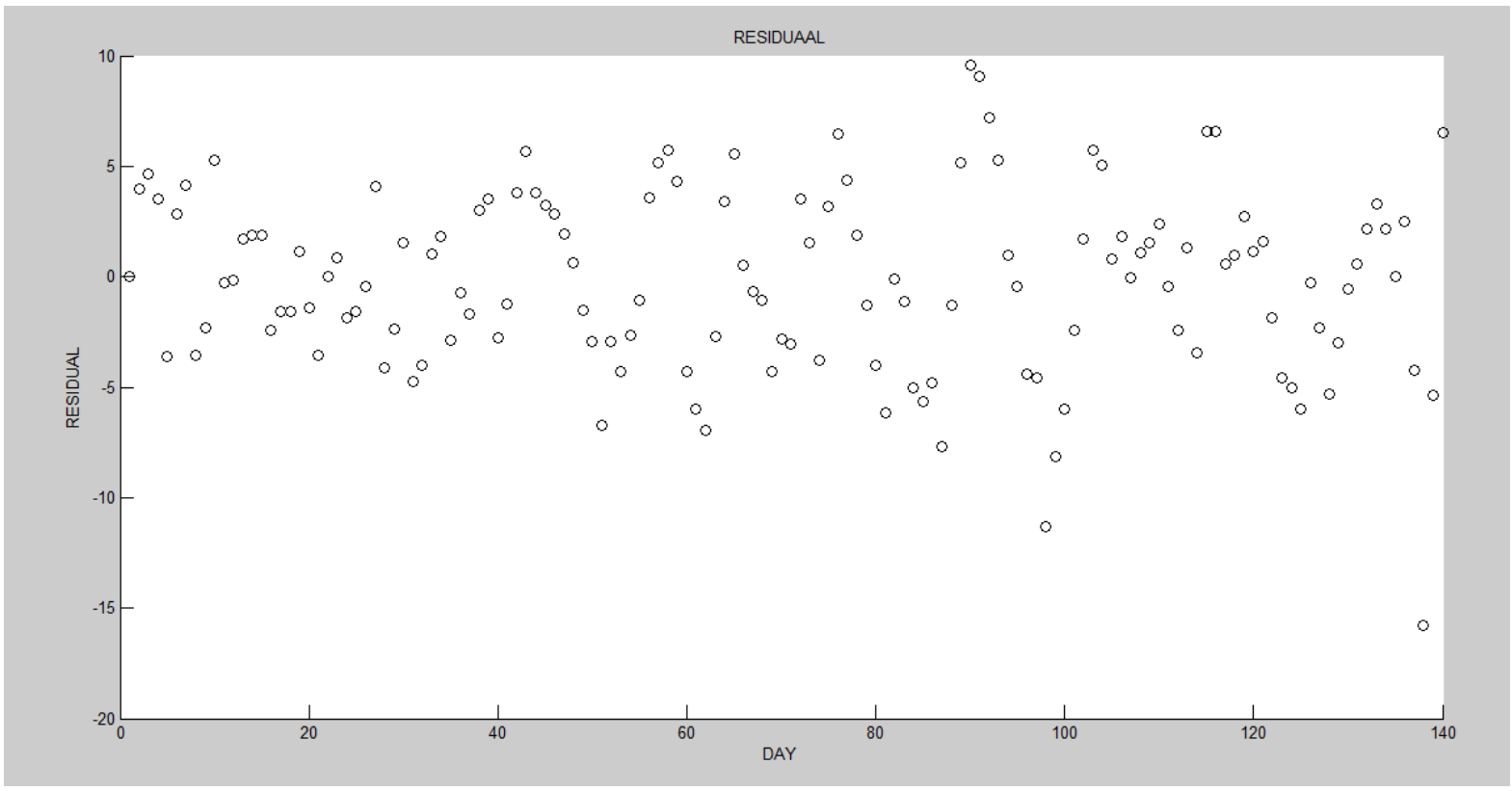

Fig.9: Scatter Plot: $\mathrm{SpO}_{2}$ time series data-scatter plot. Calculation of residue. 


\section{Research Results and Future Works}

Thus, the time series data for peripheral oxygen saturation is obtained from the patient's record and the data is decomposed into various trend components [5]. These trend components are greatly useful in estimating the condition of the patient over various time intervals. The result of this analysis can be transmitted via message to the clinical attendant of that patient.

1. The estimated residue of the time series data can be used to detect the drastic deterioration in the condition of the patient. If the change in condition is more than $50 \%$ of the previous value, alert message can be transmitted to the clinical attendant of the patient.

2. Since this is only a prototype of the analysis part, real time implementation of the system can be done after testing the system with a number of more patient records.

3. The transmission of the condition of the patient can be made on a daily basis by transmitting the condition (either good or bad) irrespective of the criticality of the patient based on [6][7].

\section{References}

[1] Alexander Schacht, Robert Wierschke, Martin Wolf, Martin von L"owis and Andreas Polze ,"Live Streaming of Medical Data The Fontane Architecture for Remote Patient Monitoring and its Experimental Evaluation." 14th IEEE International Symposium on Object/Component/Service-Oriented Real-Time Distributed Computing Workshops, 2011.

[2] S. Charbonnier and S. Gentil, "A trend-based alarm system to improve patient monitoring in intensive care units," Control Eng. Pract., vol. 15, no. 9, pp. 1039-1050, 2007.

[3] C.D.Hanningand, J.M.Alexander-Williams, "Pulseoximetry: A practical review," Brit. Med. J., vol. 311, no. 7001, pp. 367-370, 1995.

[4] Liang-Hung Wang, Yi-Mao Hsiao, Xue-Qin Xie, and Shuenn-Yuh Lee, “An Outdoor Intelligent Healthcare Monitoring Device for the Elderly" IEEE Transactions on Consumer Electronics, Vol. 62, No. 2, May 2016

[5] Malcolm Clarke, HulyaGokalp, Joanna Fursse, and Russell W Jones, "Dynamic Threshold Analysis of Daily Oxygen Saturation for Improved Management of COPD Patients"IEEE journal of biomedical and health informatics, vol. 00, no. 00, 2015.

[6] M Clarke, "The need for an integrated approach to remote monitoring of physiological data and activity data," J. Telemed. Telecare, vol. 20, no. 3, pp. 159-160, Apr. 2014.

[7] Takuo Aoyagi," Pulse oximetry: Its origin and development",Engineering in Medicine and Biology Society, 1992 14th Annual International Conference of the IEEE, 02 May 2011.

[8] S.Takatani," A non-invasive Reflactance Pulse Oximeter Sensor" Engineering in Medicine and Biology Society, 1991. Vol.13: 1991, Proceedings of the Annual International Conference of the IEEE, 06 August 2002. 